Tines de mayo de 1974. El manicomio de Trieste en Italia organiza el 1 primer concierto que hace encontrar, por primera vez dentro los muros, 1 más de mil personas de la ciudad de Trieste con otros tantos de "la ciudad de los locos". Rossetta Lojacono, que entonces tendría unos cincuenta años de los cuales llevaba más de 20 internada, subió al escenario cuando el espectáculo no había todavía iniciado y empezó a tocar su acordeón con tanta cancha, casi profesional, que Ornette Coleman dijo: "Ésto es música! Sigámosla...", y así empezó su concierto. Era una canción popular italiana. Dijo después, cuando le preguntaron sobre el hecho totalmente espontáneo, "no tenía la minima idea de quién iba a ser el público en ese hospital, nos encontramos con gente de todo tipo que no bubiera podido decir, mirándolos a la cara, éste es enfermo, éste no....”.

Lo que nadie puede negar es que Ornette Coleman intuyó que esa decisión lo iba a dejar protagonista del futuro, y eso era algo que sabía hacer muy bien, adelantarse a su tiempo. (Claudio Cúneo, 12 de junio 2015).
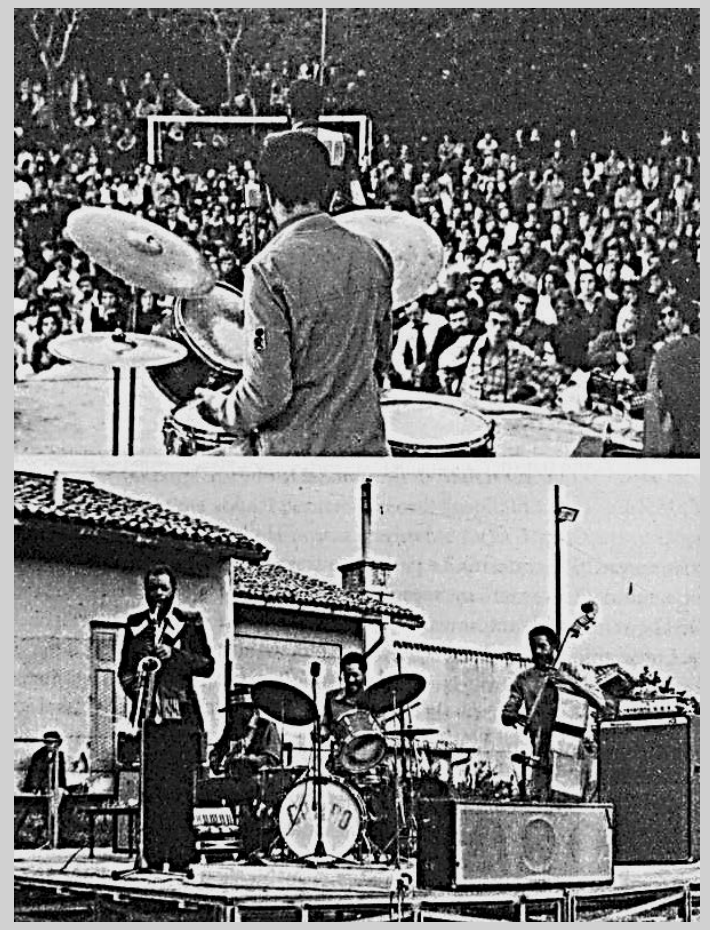

Foto Archivo Diarios Italianos 


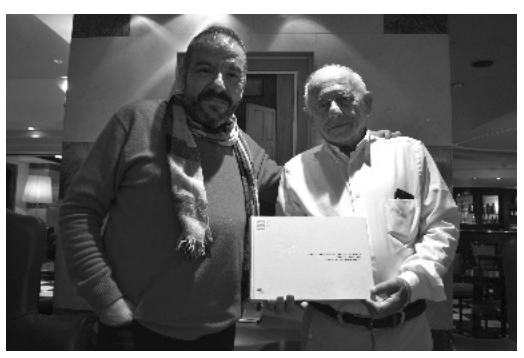

\section{Entrevista a Franco Rotelli}

(Buenos Aires, agosto 2018)

Claudio Cúneo: Franco, sos protagonista de una historia que alguna vez definieron de "locos" y "sanos", de hombres y mujeres, de jóvenes y ancianos, de psiquiatras, enfermeros y voluntarios. Una historia de gente pobre, de dolor y sufrimiento, pero también de libertad recuperada, de coraje y optimismo, de felicidad y ganas de vivir. En el fondo, una historia que cuenta las ganas de cambiar el mundo. En el 2015 escribiste el libro "L'istituzione inventata" Almanacco 1971-2010, un libro bellísimo que cuenta una historia muy conocida, con infinidad de detalles no tan conocidos y que fue enseguida un enorme éxito editorial en Italia. Lo definiste un libro "pedante" porque es lo que sucede cuando alguien quiere reconocer la “banalidad del bien”. En la presentación, en parte explicás por qué creíste útil este libro, aún. Decís que el mundo de la Psiquiatría que ves cuando viajás por el mundo sigue siendo feo, muy feo, y que el de la salud tendría que ser mucho, pero mucho mejor. Por esta doble afirmación, y porque aseverás que la historia de tu libro es inevitablemente circular, Barquitos Pintados te pide, aprovechando tu disponibilidad en esta tarde gris de Buenos Aires, que le des para nosotros, una vuelta más.

Franco Rotelli: Muchas gracias, Claudio, por esta oportunidad. Se las debía, porque nos íbamos a encontrar en Italia en el 2015 y no pudimos. Este libro se llama "La institución inventada" y está inspirado en el libro de Franco Basaglia cuyo título es "La institución negada". En ese libro Basaglia contaba la historia de Gorizia, la historia de un grupo de psiquiatras que pusieron radicalmente en discusión el hospital psiquiátrico y sus reglas. Negaron la bondad de esas instituciones y al mismo tiempo denunciaron que esa institución era el fruto de la psiquiatría, no nacían de un repollo. Es necesario aclarar que esa institución era la expresión de la psiquiatría. Denunciando las condiciones del manicomio se denunciaba a la psiquiatría entera, como madre de este hijo maligno. Esta denuncia tuvo una resonancia increíble porque estaba bien documentada, con fotografías, documentales sobre las condiciones de vida de los manicomios que fueron difundidas por la televisión italiana en un modo muy eficaz. El libro "La institución negada" denunciaba estas condiciones e indicaba lo que se estaba haciendo dentro del Hospital de Gorizia para cambiar esas condiciones en una suerte de transformación del hospital psiquiátrico en comunidad terapéutica. Basaglia se enfrentó en Gorizia con una administración pública local que era un muro, aceptaba las transformaciones en el interior del hospital psiquiátrico, pero no aceptaba que estas transformaciones salieran del Hospital Psi- 
quiátrico. Basaglia abandona Gorizia, renuncia y publica este libro "La Institución negada”. Algunos años después, por suerte, la Administración Provincial de Trieste, una ciudad cercana a Gorizia pide a Basaglia que vaya allí a afrontar el Hospital Psiquiátrico y le da carta blanca, en parte avalado por el éxito de ese libro y de toda la campaña mediática. Le dicen: "nosotros queremos un cambio radical". Toma la dirección del Hospital Psiquiátrico de Trieste en el 1971 y nace esta historia de la gradual demolición del hospital psiquiátrico que culmina en el hecho de que el Parlamento Italiano acepta en el 1978 hacer una ley que dice: "de acuerdo,pueden cerrar estos hospitales psiquiátricos porque no son la solución justa...", y acepta crear dispositivos alternativos al hospital psiquiátrico. Este libro nos cuenta este proceso y sobre todo nos cuenta cómo nace, cómo se desarrollan estas nuevas instituciones, cómo se inventan estas nuevas instituciones sustitutivas de aquellas que estaban siendo negadas en el libro precedente. ¿Qué sucedió, entonces? Se salió de la simplificación de la realidad del manicomio, se salió de la simplificación de la psiquiatría para ir a buscar la complejidad de respuestas que no tienen nada que ver con el simple psicofármaco, que no tienen nada que ver con la psicoterapia individual, con el abandono, con la invalidación del paciente psiquiátrico. Se trata de cambiar completamente de paradigma, estamos frente a ciudadanos, se trata de ciudadanos que tienen necesidades y a estas necesidades hay que afrontarlas. No podés atender solo la enfermedad, si mirás solo a la enfermedad vas a reproducir enfermedad. El tema es la persona, también su enfermedad, y aun cuando se trata de un concepto ambiguo, hasta su enfermedad podría ser modificada si tratás la persona en su contexto de vida; un ciudadano que tiene que reconstruir sus derechos se transforma en un elemento esencial de su trabajo terapéutico, rehabilitativo, curativo, emancipatorio, podés llamarlo como quieras. Es un esfuerzo por transformar en una complejidad a una simplificación. El manicomio no hacía otra cosa que sacar derechos que invalidaban, negaban, encerraban, y aquí se trataba de hacer exactamente el contrario: de validar, de enriquecer, de incluir en la comunidad, de sostener derechos, de dar respuestas articuladas a todas las necesidades de las personas. Hablo de necesidades de la vida cotidiana: casa, dinero, afectividad, trabajo, relaciones de grupo, etc. Esto tiene que hacer la política de Salud Mental. En este libro trato de contar este trabajo que pasa del manicomio a esta gama de servicios, a esta gama de posibilidades que comprenden grupos de teatro, centros de salud mental presentes en la comunidad 24/7, en el barrio, con enfermeros, psiquiatras, psicólogos que van a la casa de las personas, que no esperan que vengan al Servicio; comprende también la construcción de grupos de departamentos para pequeños grupos de personas con diferentes niveles de protección según la gravedad del problema o la necesidad, comprende la constitución de cooperativas sociales en las que se trata de incluir en el mundo del trabajo a personas que pueden todavía desarrollar algunas actividades, comprende escuelas que permitan recuperar años de escolaridad que se perdieron, laboratorios de actividad teatral, de pintura, musicales, de arte para recuperar la creatividad que existe muchas veces en modalidad más profunda en las personas con sufrimiento subjetivo. Se busca dar una respuesta al problema habitacional y es así que se van articulando todas estas respuestas, que tienen en cuenta además la particularidad del abordaje global en situaciones que requieren de un cuidado que a veces puede llevar mucho tiempo.

C.C.:¿Cuáles fueron los actos más significativos de este trabajo?

F.R.: Son muchos. Los más emblemáticos en la superación del manicomio, entre los más conocidos tenés: la construcción dentro del Hospital Psiquiátrico del gran caballo en papel ma- 
ché, Marco Cavallo, que era un caballo que existía en serio dentro del manicomio, que acarreaba un carro con las sábanas a los pabellones. Se construyó en un laboratorio teatral y artístico que funcionaba dentro de un pabellón. Siguiendo las indicaciones de los pacientes, se construye este enorme caballo que va a tomar el nombre de aquel caballo existente dentro el manicomio. Con este gran caballo, un día del año 1973, Basaglia junto a muchos médicos y pacientes, atravesaron la ciudad en un paseo por las calles que, tenés que entender sucedía en una época en que el manicomio era una institución completamente cerrada y a la que nadie podía entrar. Por el contrario, en esta ocasión se asistió a una procesión de locos que salían del manicomio y se instalaban en la plaza de la ciudad, llevando este caballo ante el estupor de toda la ciudadanía de Trieste. Otro fue el día que Franco Basaglia se hizo regalar un viaje aéreo y voló por toda la ciudad, con pacientes, con toda la resonancia mediática que estos eventos tenían, los grandes conciertos con OrnetteColleman, los espectáculos de Darío Fo y otros, que se desarrollaban dentro del manicomio y a los que se invitaba a la ciudad como un modo de demostrar que no se trataba de personas prontas a matar a todos los que pasaban por ahí. Estas fueron todas situaciones en que salíamos a la ciudad y la ciudad entraba al manicomio. Pero sobre todo, las personas empezaban a ser externadas con médicos, enfermeros, psicólogos que los acompañaban apoyándose en los Centros de Salud Mental abiertos 24/7. Se trató de luchas permanentes, todo esto hasta el 1978. Eran todas prácticas que se construían teniendo como base la ley italiana precedente que prohibía que el paciente psiquiátrico saliera del manicomio. El que salía lo hacía cometiendo un delito, era como si escapara de una cárcel, literal. El Director era responsable, como sabés, Basaglia tuvo que afrontar numerosos procesos porque sucedían estas cosas. Tuvimos problemas con la justicia, el más importante fue el nacimiento de la primer Cooperativa Social. Como en todas las instituciones totales, los pacientes trabajaban, hacían trabajos de limpieza, de jardinería, en la cocina, en la lavandería. La institución se aprovechaba de los pacientes a cambio de un paquete de cigarrillos, de algún dinero, pero prácticamente a cambio de nada. Llegados a un cierto punto del proceso, decidimos con la administración que estos trabajos fueran retribuidos, proponiendo el nacimiento de una Cooperativa Social que fuese reconocida como una empresa, que se le diera un reconocimiento jurídico. El Juzgado dijo que no, que esas eran personas incapaces y que por lo tanto no podían constituirse en Cooperativa, no podían crear una empresa, porque una empresa tiene responsabilidades jurídicas. Nos llevó dos años convencer a los Jueces que en cambio era posible, pidiendo a algunos operadores que los acompañaran en el Consejo de Administración y a algunos pacientes que decidieran ocupar esos lugares. Nació así la primer Cooperativa Social Italiana de Personas con Capacidades Diferentes, hoy hay diez mil cooperativas iguales en toda Italia. Hay muchos episodios más que fueron marcando distintos hitos, pasajes. Cuando estábamos decidiendo cerrar el manicomio, superarlo, evidentemente, el tema de la casa, el tema de dónde ir a vivir era dramático. El primer tiempo nadie nos alquilaba departamentos así que nosotros, un grupo de médicos junto a un grupo de pacientes, fuimos a ocupar un edificio de propiedad pública deshabitado. Lo ocupamos para indicar a la ciudad que teníamos necesidad de vivienda para estas personas. Pensá que el hecho fue que médicos, psicólogos, enfermeros ocupaban un edificio de propiedad pública; imagináte la paradoja y la resonancia, la amplitud mediática que tuvo esto en la ciudad y en el país. Nosotros nos ocupamos allí de construir el Estado de Bienestar porque la Psiquiatría no es una práctica adecuada si no se ocupa del bienestar general, de la vida de estas personas en 


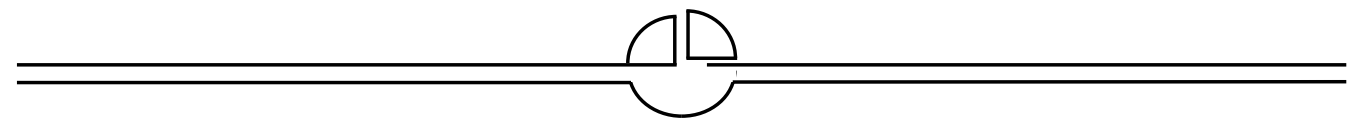

un sentido global. Después de este evento, las entidades públicas empezaron a darnos casas y hoy no hay ningún problema, esas personas lograron a través de estos nuevos reglamentos, la posibilidad de tener una casa donde vivir. Entonces: el trabajo a través de las cooperativas, las políticas sobre vivienda... Otra cosa que en Trieste sigue siendo muy importante, es la relación con el mundo: argentinos, españoles, brasileros, colaboran con Trieste, van a visitarnos, trabajan con nosotros. Hemos llevado al mundo una idea, estas experiencias. Esto nos da fuerza y nos dio energía a lo largo de los años, sobre todo en los primeros años en los que teníamos mucha necesidad de jóvenes que nos acompañaran. Todo esto para llevar adelante una lucha contra la burocracia, la inercia, contra los miedos fantásticos sobre la peligrosidad del loco... Vos sabés que eran más de 100.000 personas internadas en los hospitales psiquiátricos de Italia en los años 60 y hoy no queda ningún hospital psiquiátrico... y lo más importante es que estos 100.000 locos no destruyeron Italia.

C.C.: ...quizás fueron otros los que la destruyeron...(risas)

F.R.: Sí, tenés razón, quizás fueron otros los que la destruyeron. Bueno, esta es la historia, una historia que todavía hoy hace discutir al país. Porque la medicina condicionada por las reglas del sistema público, la cultura de las varias psicologías, psico-ciencias reductivas, no ayudan a dar respuestas articuladas, ricas a las personas, sino que por el contrario tienden a medicalizar, psicologizar, hospitalizar en lugar de ofrecer respuestas en la riqueza de la vida para que las personas puedan obtener una vida más rica. Derechos concretos en una comunidad concreta. Sigue siendo un trabajo difícil, lleno de obstáculos, de estigmas, prejuicios, de miedos y angustias que la enfermedad mental produce en las personas en el momento de una crisis, sumado a las condiciones sociales en las que viven estos ciudadanos que los colocan, muy a menudo, en una posición contractual muy débil a la hora de acceder a los servicios. Por eso es necesario seguir sosteniendo un movimiento fuerte y en esto los técnicos tienen una enorme responsabilidad en su defensa, si quieren hacer bien su trabajo. Hasta ayer, su poder no se usaba para defender el derecho de estas personas sino para defender a la sociedad de estos presuntamente sujetos peligrosos, ofreciéndose como custodios en lugar de ofrecerse como terapeutas.

C.C.: Gracias, Franco Rotelli. Por tu generosidad para con nuestra revista y gracias porque volver a contar esta historia me permite recordar que en Japón hay todavía 300.000 personas internadas en manicomios, y que acá todavía tenemos varios miles, en una situación que mejoró muchísimo gracias a la Ley de Salud Mental y que, en el 2020, cambiará todavía más. Como sabés, este país se propone cerrar todos los Hospitales Psiquiátricos, siguiendo la horma de la historia italiana que tu libro tan bien documenta. Volvé a la Argentina cuando quieras que vas a ser bienvenido y va a ser, como siempre, un enorme placer volver a darle un giro a esta historia circular, encontrándole siempre otra vuelta. Hasta la próxima. 